\title{
Impact of rotavirus vaccination on rotavirus genotype distribution and diversity in England, September 2006 to August 2016
}

Daniel Hungerford ${ }^{1,2,3,4}$, David J Allen ${ }^{3,5}$, Sameena Nawaz ${ }^{6}$, Sarah Collins ${ }^{7}$, Shamez Ladhani ${ }^{7,8}$, Roberto Vivancos ${ }^{2,3,4}$, Miren

Iturriza-Gómara ${ }^{1,3,4}$

1. The Centre for Global Vaccine Research, Institute of Infection and Global Health, University of Liverpool, Liverpool, United Kingdom

2. Field Epidemiology Services, National Infection Service, Public Health England, Liverpool, United Kingdom

3. NIHR Health Protection Research Unit in Gastrointestinal Infections, Liverpool, United Kingdom

4. NIHR Health Protection Research Unit in Emerging and Zoonotic Infections, Liverpool, United Kingdom

5. Department of Pathogen Molecular Biology, Faculty of Infectious and Tropical Diseases, London School of Hygiene and Tropical Medicine, London, United Kingdom

6. Virus Reference Department, National Infection Service, Public Health England, London, United Kingdom

7. Immunisation Department, National Infection Service, Public Health England, London, United Kingdom

8. NIHR Health Protection Research Unit in Immunisation, London School of Hygiene and Tropical Medicine, London, United Kingdom

Correspondence: Daniel Hungerford (d.hungerford@liverpool.ac.uk)

Hungerford Daniel, Allen David J, Nawaz Sameena, Collins Sarah, Ladhani Shamez, Vivancos Roberto, Iturriza-Gómara Miren. Impact of rotavirus vaccination on rotavirus genotype distribution and diversity in England, September 2006 to August 2016. Euro Surveill. 2019;24(6):pii=1700774. https://doi.org/10.2807/15607917.ES.2019.24.6.1700774

Introduction: Rotavirus vaccination with the liveattenuated monovalent (a G1P[8] human rotavirus strain) two-dose Rotarix vaccine was introduced in England in July 2013. Since then, there have been significant reductions in rotavirus gastroenteritis incidence. Aim: We assessed the vaccine's impact on rotavirus genotype distribution and diversity 3 years post-vaccine introduction. Methods: Epidemiological and microbiological data on genotyped rotavirus-positive samples between September 2006 and August 2016 were supplied by EuroRotaNet and Public Health England. Multinomial multivariable logistic regression adjusting for year, season and age was used to quantify changes in genotype prevalence in the vaccine period. Genotype diversity was measured using the Shannon's index (H') and Simpson's index of diversity (D). Results: We analysed genotypes from 8,044 faecal samples. In the pre-vaccine era, $\mathrm{G}_{1} \mathrm{P}[8]$ was most prevalent, ranging from $39 \%(411 / 1,057)$ to $74 \%(527 / 709)$ per year. In the vaccine era, $\mathrm{G}_{1} \mathrm{P}[8]$ prevalence declined each season ( $35 \%, 231 / 654 ; 12 \%, 154 / 1,257$; $5 \%, 34 / 726)$ and genotype diversity increased significantly in 6-59 months old children ( $H^{\prime} \mathrm{p}<0.001$ : D p 0.001). In multinomial analysis, G2P[4] (adjusted multinomial odds ratio (aMOR): 9.51; 95\% confidence interval (Cl): 7.02-12.90), G3P[8] (aMOR: 2.83; 95\% Cl: 2.17-3.81), G12P[8] (aMOR:2.46; 95\% Cl: 1.62-3.73) and $\mathrm{G}_{4} \mathrm{P}[8]$ (aMOR:1.42; $\left.95 \% \mathrm{Cl}: 1.02-1.96\right)$ significantly increased relative to $\mathrm{G}_{1} \mathrm{P}[8]$. Conclusions: In the context of reduced rotavirus disease incidence, genotype diversity has increased, with a relative change in the dominant genotype from $\mathrm{G}_{1} \mathrm{P}[8]$ to $\mathrm{G}_{2} \mathrm{P}[4]$ after vaccine introduction. These changes will need continued surveillance as the number and age of vaccinated birth cohorts increase in the future.

\section{Introduction}

Prior to the introduction of rotavirus vaccination, Group A rotaviruses were the most common cause of severe childhood diarrhoea globally, resulting in over 450,000 deaths in children aged under 5 years [1]. In England and Wales, rotavirus accounted for ca 80,000 general practice (GP) consultations and was responsible for $45 \%$ of acute gastroenteritis hospital admissions [2,3]. In July 2013, the United Kingdom (UK) introduced the monovalent ( $\mathrm{G}_{1} \mathrm{P}[8]$ ) live-attenuated, two-dose oral human vaccine (Rotarix, GlaxoSmithKline Biologicals, Rixensart, Belgium) into the routine childhood immunisation schedule at 8 and 12 weeks of age [4]. Rotavirus vaccine uptake increased rapidly to over $90 \%$ for one dose and has remained consistently high; in July 2016 , vaccine uptake was over $94 \%$ for one dose and over $90 \%$ for the recommended two-dose schedule [5]. In high-income countries, efficacy of the monovalent G1P[8] Rotarix vaccine against severe rotavirus gastroenteritis was estimated at over $85 \%$ and further trials have shown that it is efficacious against multiple rotavirus strains $[6,7]$. Since vaccine implementation in the UK, data from laboratory reports, hospital admissions and GP consultations have shown a significant reduction in the incidence of rotavirus gastroenteritis in vaccine eligible children (children born since 01 May 
2013), older vaccine ineligible children and adults $(\geq 18$ years) [8-11].

Group A rotaviruses are defined by the middle capsid antigen and further classified into $G$ and $P$ types based on serological and genetic characterisation of two outer viral proteins, the VP7 (a glycoprotein) and the VP4 (a protease sensitive protein), respectively. Furthermore, whole genome sequencing has allowed rotavirus strains to be classified according to genotype constellations by using a common genetic backbone constellation (defined by nine of eleven gene seg. ments, excluding VP7 and VP4). Among human group $\mathrm{A}$ rotaviruses there are two common genotype constellations: Wa-like and DS-1 like [12].

Prior to introduction of rotavirus vaccination, genotype $\mathrm{G}_{1} \mathrm{P}[8]$ was the predominant circulating rotavirus genotype in the UK [13-17]. In England, like in most countries that have recently introduced rotavirus vaccination, monitoring the impact of the rotavirus vaccine includes the assessment of changes in healthcare utilisation and disease incidence, as well as monitoring genotype distribution. Rotavirus genotype surveillance has been undertaken systematically pre- and post-vaccine introduction in the context of the European Rotavirus Network, EuroRotaNet, which was established in January 2007 and has 14 member countries, including the UK $[18,19]$. Each member country conducts rotavirus genotype surveillance; laboratory and epidemiological data are also collated within the network to generate comprehensive information on the rotavirus genotypes co-circulating throughout Europe. This multi-centre and data-sharing network approach allows widespread monitoring of circulating rotavirus genotypes in order to identify: (i) possible vaccine-induced emergence of antibody escape mutants; (ii) possible emergence of non-vaccine genotypes; and (iii) possible emergence of reassortants between vaccine-type and naturally circulating wild-type strains.

Because England has one of the largest historical rotavirus genotype databases in Europe, dating back to the late $1990 \mathrm{~s}[13,16,17]$, and has used consistent laboratory diagnostics and genotyping methods, it is an ideal study location for monitoring any changes in genotype distribution post-vaccine introduction. This study evaluates the impact of routine childhood rotavirus vaccination on relative changes in rotavirus genotype distribution and diversity in England.

\section{Methods}

\section{Study area and samples}

The study area was England, covering all regions. Public Health England (PHE), Colindale, receives electronic laboratory reports of laboratory-confirmed rotavirus infections from diagnostic laboratories in England and Wales. In this study, samples included rotavirus-positive faecal samples from mostly sporadic gastroenteritis cases (if associated with outbreaks, only a single sample per outbreak) submitted for routine diagnostic testing and genotyped at the PHE Virus Reference Department (VRD) using standardised G and P typing methods $[14,20]$.

Prior to vaccine introduction, selection of rotaviruspositive samples for genotyping was passive. As part of EuroRotaNet, regional laboratories in England submitted any rotavirus-positive residual samples to the VRD. Although submission of samples to VRD was not actively followed-up, at a country level the minimum target number of specimens included for genotyping would enable detection of rotavirus genotypes with a prevalence of $\geq 1 \%$ [19].

When the infant rotavirus vaccination programme was introduced in July 2013, the Immunisation Department at PHE initiated active surveillance for rotavirus. Hospital laboratories across England and Wales were actively requested to submit all rotavirus-positive samples in vaccine-eligible children to the PHE VRD for confirmation and genotyping. After the introduction of rotavirus vaccination $G_{1} P[8]$ vaccine-derived strains were defined on the basis of the sequences of the VP4 and $\mathrm{VP} 7$ encoding genes displaying highest homology with Rotarix sequences and/or through the detection of the Rotarix strain using a published and validated qRT$P C R$ assay which specifically targets the non-structural protein 2 (NSP2) gene of the Rotarix strain [21].

\section{Data}

The sampling methods used mean that the data in this study do not represent rotavirus incidence but allow us to assess relative changes in genotype distributions. Details on case age, region, specimen collection date and rotavirus genotyping results for specimens collected between September 2006 and August 2016, were included in this study. Data on cases' sex were incomplete, so we were unable to include these in the analyses however, previous analysis of EuroRotaNet data has shown that there is no difference in genotype distribution by sex [19].

While EuroRotaNet was established in January 2007, data dating back to September 2006 were retrospectively collected by this network. Thus data from September 2006 to December 2012 are held by EuroRotaNet and, and from January 2013 to August 2016, jointly by EuroRotaNet and PHE. In order to maintain complete surveillance years (September to August) for analysis, the pre-vaccine period was defined as September 2006 to August 2013 and the vaccine period was defined as September 2013 to August 2016.

Age groups of cases ( 6 months, 6-11 months, 12-23 months, 2-4 years and $\geq 5$ years) were constructed using date of birth and date of specimen submission. Genotyped rotavirus strains were categorised according to their possible evolutionary origin [14]. A derived binary 'season' variable was constructed to indicate whether a rotavirus case occurred during the 
Number of rotavirus specimens collected in the pre-vaccine era and the vaccine era, by genotype and age group, England, September 2006-August $2016(n=8,044)$

\begin{tabular}{|c|c|c|c|c|c|c|c|c|c|c|}
\hline \multirow{3}{*}{$\begin{array}{l}\text { Characteristic } \\
\text { Genotype }\end{array}$} & \multirow{2}{*}{\multicolumn{2}{|c|}{$\begin{array}{c}\text { Pre-vaccine era } \\
\text { Sep } 2006 \text {-Aug } 2013 \\
(n=5,407)\end{array}$}} & \multicolumn{8}{|c|}{ Vaccine era } \\
\hline & & & \multicolumn{2}{|c|}{$\begin{array}{c}\text { Sep } 2013-\text { Aug } 2014 \\
(n=654)\end{array}$} & \multicolumn{2}{|c|}{$\begin{array}{c}\text { Sep } 2014-\text { Aug } \\
2015 \\
(n=1,257)\end{array}$} & \multicolumn{2}{|c|}{$\begin{array}{c}\text { Sep } 2015 \text {-Aug } 2016 \\
(n=726)\end{array}$} & \multicolumn{2}{|c|}{$\begin{array}{c}\text { Sep } 2013-\text { Aug } \\
2016 \\
(n=2,637)\end{array}$} \\
\hline & $\mathrm{n}$ & $\%$ & $\mathbf{n}$ & $\%$ & $n$ & $\%$ & $\mathrm{n}$ & $\%$ & $n$ & $\%$ \\
\hline $\mathrm{G}_{1} \mathrm{P}[8]$ & 2,987 & 55 & 231 & 35 & 154 & 12 & 34 & 5 & 419 & 16 \\
\hline $\mathrm{G}_{2} \mathrm{P}[4]$ & 397 & 7 & 28 & 4 & 345 & 27 & 325 & 45 & 698 & 26 \\
\hline $\mathrm{G}_{3} \mathrm{P}[8]$ & 527 & 10 & 190 & 29 & 101 & 8 & 61 & 8 & 352 & 14 \\
\hline G4P[8] & 368 & 7 & 53 & 8 & 103 & 8 & 21 & 3 & 177 & 7 \\
\hline G8P[4] & 118 & 2 & 0 & 0 & 0 & 0 & 2 & 0 & 2 & 0 \\
\hline G9P[8] & 597 & 11 & 46 & 7 & 191 & 15 & 134 & 18 & 371 & 14 \\
\hline G12P[8] & 122 & 2 & 19 & 3 & 242 & 19 & 17 & 2 & 278 & 11 \\
\hline G1P[8]-unknown ${ }^{a}$ & 0 & 0 & 0 & 0 & 0 & 0 & 3 & 0 & 3 & 0 \\
\hline $\mathrm{G} 1 \mathrm{P}[8]-\mathrm{VD}$ & 0 & 0 & 64 & 10 & 84 & 7 & 67 & 9 & 215 & 8 \\
\hline Other ${ }^{\mathrm{b}}$ & 91 & 2 & 6 & 1 & 27 & 2 & 32 & 4 & 65 & 2 \\
\hline Mixed $^{c}$ and untypable & 200 & 4 & 17 & 3 & 10 & 1 & 30 & 4 & 57 & 2 \\
\hline Age group ${ }^{d}$ & $\mathrm{n}$ & $\%$ & $n$ & $\%$ & $n$ & $\%$ & $n$ & $\%$ & $n$ & $\%$ \\
\hline$<6$ months & 577 & 11 & 169 & 25 & 233 & 19 & 102 & 15 & 504 & 20 \\
\hline 6-11 months & 1,194 & 24 & 105 & 16 & 124 & 9 & 61 & 9 & 290 & 11 \\
\hline 12-23 months & 1,851 & 37 & 261 & 40 & 407 & 34 & 234 & 34 & 902 & 35 \\
\hline $2-4$ years & 940 & 19 & 81 & 12 & 369 & 34 & 238 & 35 & 688 & 27 \\
\hline$\geq 5$ years & 464 & 9 & 37 & 6 & 79 & 7 & 52 & 8 & 168 & 7 \\
\hline
\end{tabular}

VD: vaccine-derived.

${ }^{a} G_{1} P[8]$-unknown: unknown whether these strains are wild-type $G_{1} P[8]$ or VD.

b 'Other' refers to rarer genotypes, which contributed $<1 \%$ of samples over the study period.

'Mixed: more than one genotype found in an individual sample.

${ }^{d}$ There were 466 specimens with unknown case age. Percentages are calculated from total specimens with known case age.

pre-vaccine introduction peak rotavirus season (calendar week 1 to week 25) or the non-peak rotavirus season (calendar week 26 to week 52) according to the date of specimen collection [17].

For the purposes of statistical analyses (apart from genotype diversity), because of the large number of genotypes, any single genotype which contributed $<1 \%$ of samples over the study period was classified into a generic 'other' category for 'rarer' genotypes. A separate grouping 'mixed or untypable' was established for any mixed (more than one genotype found in one individual sample) or partially typed strains.

\section{Vaccination status}

As part of the enhanced surveillance, the general practitioner of each rotavirus-positive infant in the vaccine-eligible age group (born since 01 May 2013) was contacted to establish their rotavirus vaccine history. For this study, the vaccine history was assessed for samples with a $\mathrm{G}_{1} \mathrm{P}[8]$ vaccine-derived strain. The vaccination history, included number of rotavirus vaccine doses received and date of vaccination.

\section{Data analysis}

Descriptive analysis

Data analyses were performed using $R$ version 3.3.2 [22]. The distribution of rotavirus G- and P-genotypes was tabulated in respect to age group, year, and period (pre- or post-vaccine introduction). Differences between continuous variables were tested using Student's t-test or Wilcoxon rank-sum test if not normally distributed and chi-squared-test or Fisher's exact test for categorical variables. Any $\mathrm{G}_{1} \mathrm{P}[8]$ vaccine-derived samples were excluded from the statistical analyses under the assumption that they were not the disease causative agent.

Genotype differences pre- and post-vaccine introduction To investigate relative statistical differences in circulating genotypes pre- and post-vaccine introduction, we fitted multinomial logistic regression models, with genotype as the outcome and $\mathrm{G}_{1} \mathrm{P}[8]$ as the baseline genotype. Model fitting was based on season, surveillance year and age group; these terms were identified a priori [17]. The model was first run as a univariable analysis including only the binary vaccine period variable 


\section{FIGURE 1}

Number of rotavirus specimens typed per month and surveillance year, England, September 2006-August 2016 $(\mathrm{n}=8,044)$

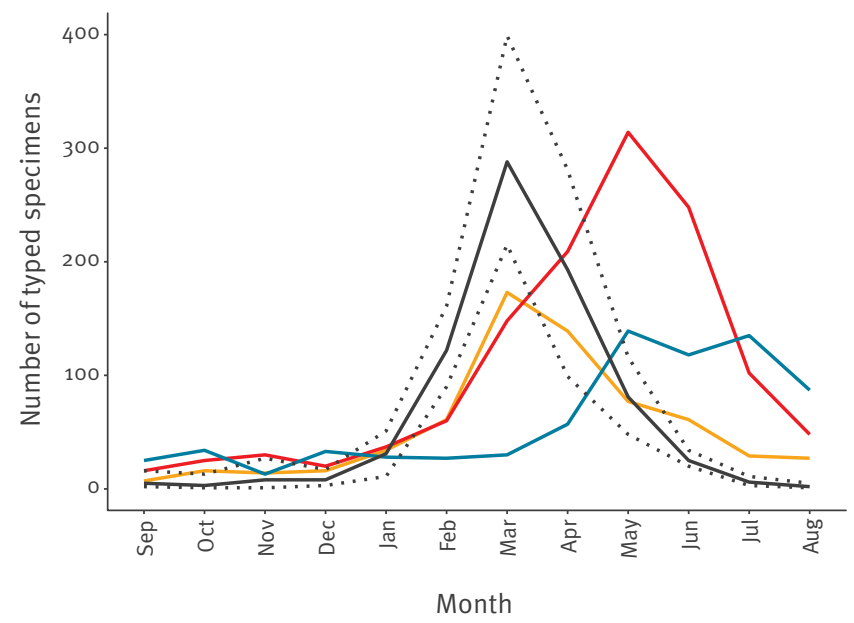

Surveillance year

$$
\begin{array}{ll}
-2013 / 14 & \text { - Pre-vaccine mean } \\
-2014 / 15 & \ldots \text { Pre-vaccine minimum } \\
-2015 / 16 & \ldots \text { Pre-vaccine maximum }
\end{array}
$$

Figure is not representative of rotavirus disease incidence.

for before or after vaccine introduction. Age-stratified models were also run; with genotype as the outcome variable, and vaccine period, season and surveillance year as covariates. Multinomial odds ratios (MOR), $95 \%$ confidence intervals $(\mathrm{Cl})$ and the associated $\mathrm{p}$ values were calculated from the Wald test. Results were considered significant at $p<0.05$.

Rotavirus genotype diversity was compared using two established biodiversity indices, Simpson's index of diversity (D) and Shannon's index (H) [23]. All single typed rotavirus genotypes were included in the analysis. Simpson's index of diversity (D) has a range between $\mathrm{o}$ and 1 (maximum diversity) and represents the probability that two randomly chosen rotavirus genotypes will have different $G$ and $P$ types and is calculated as $1-\lambda$, where $\lambda=\Sigma($ piz) and pi is the proportional abundance of a genotype i. Shannon's index $\left(H^{\prime}\right)$ ranges from $O$ (no diversity) to $Y$ (maximum) and quantifies the uncertainty in predicting the rotavirus genotype identity of an individual sample that is taken at random from the dataset and is calculated as $\mathrm{H}^{\prime}$ $=-\Sigma(\mathrm{pi} \times \ln (\mathrm{pi}))[17]$. Confidence intervals were estimated using bootstrap resampling methodology and differences by age group, pre- and post-vaccine introduction were compared using $p$ values generated from $z$-scores during bootstrap analysis. The $\mathrm{R}$ packages 'vegan' and 'boot' were used for the analysis of genotype biodiversity $[24,25]$.

\section{Results}

Descriptive statistics

In England a total of 8,044 rotavirus-positive specimens were genotyped between September 2006 and August 2016. Six per cent of specimens $(n=466)$ had no detail on case age, and most of these were from $2007 / 08(n=209 / 737 ; 28 \%)$ and 2011/12 $(n=137 / 732$; $19 \%)$. The case age ranged from « 1 month to 104 years and the majority of specimens were from children aged $<5$ years $(92 \% ; 6,945 / 7,578)$, with most specimens $(56 \% ; 4,237 / 7,578)$ from children aged 6-23 months (Table). Prior to vaccine introduction, the rotavirus season consistently occurred between January and May, with the peak occurring during March. After the introduction of rotavirus vaccination, the seasons became less pronounced; in the first surveillance year post-vaccine introduction (2013/14), the peak occurred in March, but in 2014/15 and 2016/17 the peak was in May and season lasted longer into July (Figure 1).

\section{Genotype distribution}

In the pre-vaccine period (September 2006 to August 2013), G1P[8] was the overall predominant genotype $(55 \%)$, ranging from $39 \%(411 / 1,057)$ to $74 \%(527 / 709)$ per year (Table). In cases aged 5 years and older, G1P[8] was less dominant than in younger age groups, representing 34\% (156/464) of all specimens genotyped, while genotypes G2P[4] (16\%; 73/464) and G8P[4] (8\%; 39/464) were more prevalent (Figure 2).

In the first surveillance year of the vaccine era (2013/14), G1P[8]-type viruses appeared to become less dominant overall, accounting for $35 \%$ of genotyped samples; in the same period, G3P[8] accounted for $29 \%$ (Table). In surveillance years two (2014/15) and three (2015/16) of the vaccine era, $\mathrm{G}_{2} \mathrm{P}[4]$-type viruses accounted for the highest proportion of strains ( $27 \%$ and $45 \%$, respectively). In these two surveillance years wild-type G1P[8] viruses seemed to become less prevalent, accounting for $12 \%$ of genotyped specimens in $2014 / 15$ and $5 \%$ in $2015 / 16$. Also, in 2014/15, G12P[8]-type viruses represented $19 \%$ of samples, compared with $2 \%$ in the prevaccine period.

In the pre-vaccine period $\mathrm{G}_{1} \mathrm{P}[8]$ was dominant in all children under 5 years of age (Figure 2). In the vaccine period, the distribution of rotavirus genotypes in infants younger than 6 months of age was different to the other age groups; wild-type $\mathrm{G}_{1} \mathrm{P}[8]$ viruses were present in $28 \%(141 / 504)$ of cases and vaccinederived $\mathrm{G}_{1} \mathrm{P}[8]$ strains were detected in 38\% (193/504) of cases (Figure2). G1P[8] was detected less frequently as age increased, it was detected in $18 \%(51 / 290)$ of 6-11 month olds, $14 \%$ (126/902) of $12-23$ month olds, $11 \%(78 / 688)$ of $2-4$ year olds, and $9 \%(15 / 168)$ of $\geq 5$ year-olds. The change in strain distribution between the pre-vaccine and vaccine era for all ages was significant when excluding vaccine-derived $\mathrm{G}_{1} \mathrm{P}[8]$ strains (chi-squared $=1,509$, degrees of freedom $=8 ; p<0.001$ ). 


\section{FIGURE 2}

Genotype proportions of typed rotavirus specimens by surveillance year and age group, England, September 2006-August $2016(n=8,044)$

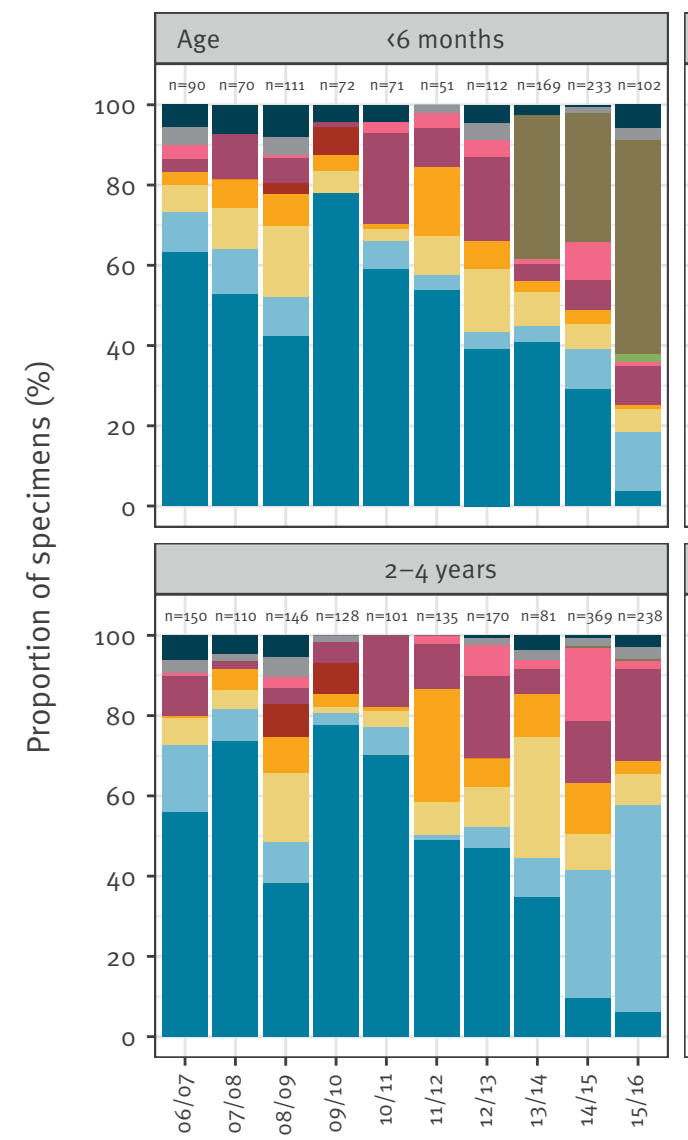

$\begin{array}{ll}\mathrm{G}_{1} \mathrm{P}[8] & \mathrm{G}_{2} \mathrm{P}[4] \\ \mathrm{G}_{12} \mathrm{P}[8] & \mathrm{G} 1 \mathrm{P}[8]-\mathrm{UK}-\text { unknown }\end{array}$
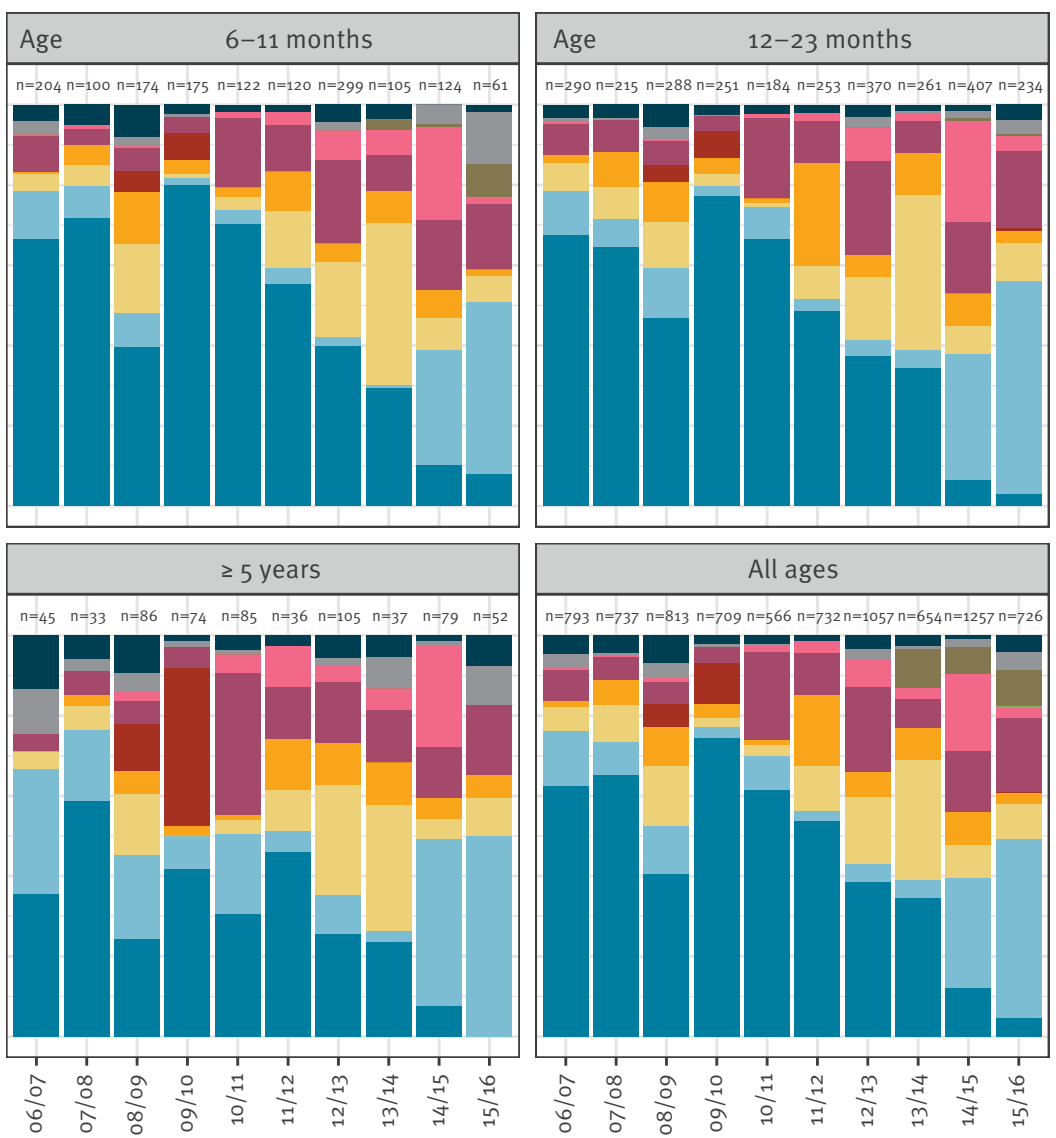

Surveillance year

$$
\mathrm{G}_{3} \mathrm{P}[8]-\mathrm{G}_{4} \mathrm{P}[8]
$$$$
\text { G8P[4] }
$$

- Mixed` and untypable

UK: United Kingdom; VD: vaccine derived.

${ }^{a} \mathrm{G}_{1} \mathrm{P}[8]-$-unknown: unknown whether these strains are wild-type $\mathrm{G}_{1} \mathrm{P}[8]$ or VD.

b 'Other' refers to rarer genotypes which contributed $<1 \%$ of samples over the study period.

c Mixed: more than one genotype found in an individual sample.

There were 466 specimens with unknown case age. Percentages are calculated from total specimens with known case age. The genotyping analyses' results are indicated by different colours, as explained in the legend.

\section{Vaccine-derived strains}

After rotavirus vaccine introduction in the UK in July 2013 there were $215 \mathrm{G} 1 \mathrm{P}[8]$ vaccine-derived strains detected.

Of the 208 with an age recorded, 92\% ( $n=191 / 208)$ were detected in infants aged 2-5 months of age, who would have been eligible for immunisation. Among these cases, six were unvaccinated infants, vaccine status was unknown for three and five were vaccinated after the sample collection date. The remaining $(n=177)$ were vaccinated with a median time between specimen date and most recent rotavirus vaccine dose of 13 days (interquartile range (IQR): 7-23) and a maximum of 154 days.

There were 12 instances where vaccine-derived $\mathrm{G}_{1} \mathrm{P}[8]$ strains were found in specimens from children eligible for vaccination but older than 6 months of age when the sample was taken. Of these, one sample had come from an unvaccinated patient and one from a non-UK resident; the remaining 10 were from patients who had received at least one dose of rotavirus vaccine between 83 days and 420 days before the sample collection date. 
A further two cases were aged 22 months of age; one had received the first dose of rotavirus vaccine 5 days before the date of sample collection and the other case had not received any vaccine doses at the time of sampling.

Three cases, aged 23 months, 3 years and 4 years were born before vaccine introduction and therefore, would have been too old for the UK rotavirus immunisation programme. Vaccine status for a 23 month-old and 4 year-old could not be followed-up, but the 3 year-old had received the second dose of the rotavirus vaccine 2 days before the date of the sample.

\section{Age}

In the pre-vaccine period, the majority of samples referred were from children aged 6-23 months (61\%; $3,045 / 5,026)$. In the vaccine era, when excluding $\mathrm{G}_{1} \mathrm{P}[8]$ vaccine-derived cases, most samples were from children aged $12-59$ months $(68 \% ; 1,584 / 2,344)$. In the pre-vaccine era, the median age was 13 months (IQR: 9-24) compared with 19 months (IQR: 11-29) in the vaccine period $(W=572,300 ; p<0.001)$.

\section{Multinomial logistic regression}

Although G8P[4] was detected in more than $1 \%$ of samples across the study period, for the multinomial regression analyses, G8P[4] was classified as a rare genotype because it was only detected in 2008/09 $(n=45) ; 2009 / 10(n=73)$ and 2015/16 $(n=2)$. We also excluded $\mathrm{G}_{1} \mathrm{P}[8]$ vaccine-derived strains from the models, under the assumption that the majority were detected in vaccinated infants and were, therefore, not the causative agent of the gastroenteritis symptoms. When adjusting for surveillance year, season and age group, the adjusted multinomial odds ratios (aMOR) of infection caused by the following genotypes: $\mathrm{G}_{2} \mathrm{P}[4]$ (aMOR: 9.51; 95\% Cl: 7.02-12.90; p<0.001); G3P[8] (aMOR:2.83; 95\% Cl: 2.17-3.81; p<0.001); G12P[8] (aMOR: $2.46 ; 95 \% \mathrm{Cl}: 1.62-3.73 ; \mathrm{p}<0.001)$; mixed and untypable (aMOR: $2.00 ; 95 \% \mathrm{Cl}: 1.20-3.36 ; \mathrm{p}<0.01$ ); and other rarer (aMOR: $2.6 ; 95 \% \mathrm{Cl}: 1.63-4.17 ; p<0.001$ ); G4P[8] (aMOR:1.42; 95\% Cl: 1.02-1.96; $\mathrm{p}=0.03$ ), were higher in the vaccine era relative to wild-type $G_{1} P[8]$, while cases due to G9P[8] (aMOR:1.06; 95\% Cl: 0.811.38; $p=0.661$ ) were unchanged (Figure 3; full model). In age-stratified analysis, across all age groups $\mathrm{G}_{2} \mathrm{P}[4]$ became significantly more prevalent in the vaccine era relative to wild-type $\mathrm{G}_{1} \mathrm{P}[8]$ (Figure 3). In infants younger than 6 months of age, G2P[4] (aMOR:3.04; $95 \% \mathrm{Cl}: 1.18-7.88 ; p=0.02)$ was the only genotype that was significantly more prevalent in the vaccine era relative to wild-type $\mathrm{G}_{1} \mathrm{P}[8]$.

\section{Genotype diversity}

There were 30 different single rotavirus genotypes (G10P[4], G12P[10], G12P[4], G12P[6], G12P[8], G1P[4], $G_{1} P[5], G_{1} P[6], G_{1} P[8], G_{2} P[10], G_{2} P[4], G_{2} P[6], G_{2} P[8]$, $\mathrm{G}_{2} P[9], \mathrm{G}_{3} P[14], \mathrm{G}_{3} P[3], \mathrm{G}_{3} P[4], \mathrm{G}_{3} P[6], \mathrm{G}_{3} P[8], \mathrm{G}_{3} P[9]$, G4P[4], G4P[8], G6P[14], G6P[9], G8P[14], G8P[4], G8P[8], G9P[4], G9P[6], G9P[8]) identified between
September 2006 and August 2016. Genotype diversity increased significantly in the vaccine era compared with the pre-vaccine era (Figure 4), and variation across the age groups was observed. There were significant increases in the genotype diversity among infants aged 6-11 months ( $\left.H^{\prime}: p<0.001 ; D: p<0.001\right)$; children aged $12-23$ months ( $\left.H^{\prime}: p<0.001 ; D: p<0.001\right)$ and children 24-59 months of age ( $\left.H^{\prime}: p<0.001 ; D: p<0.001\right)$. However, there was no change in genotype diversity among cases aged 5 years and older $\left(H^{\prime}: p=0.477\right.$; $D$ : $p=0.471$ ). An increase in genotype diversity was only observed in infants 16 months of age when using the Simpsons index ( $D: p=0.024)$.

In the pre-vaccine era, genotype diversity was significantly higher in individuals aged 5 years and older than in any other age group but this is no longer true after rotavirus vaccine introduction, genotype diversity was comparable across those aged 6-11 months; $12-23$ months, 24-59 months and those aged 5 years and older. Furthermore, genotype diversity in all of these groups was significantly higher than that in infants aged less than 6 months (for all comparison ages, $H^{\prime}$ : p 0.001 and D': p po.001).

\section{Discussion}

There has been a change in rotavirus genotype distribution and diversity in England following the introduction of rotavirus vaccination for all infants in 2013. In the context of very high rotavirus vaccine uptake (ca 94\%) [5], there has been both an increase in the genotype diversity among symptomatic cases and a change in the dominant circulating genotype from $\mathrm{G}_{1} \mathrm{P}[8]$ in the pre-vaccine era to $\mathrm{G}_{2} \mathrm{P}[4]$ in the vaccine era.

$\mathrm{G}_{2} \mathrm{P}$ [4] genotypes have been associated with outbreaks in older children (11-12 years) and adults in the absence of rotavirus vaccination or shortly after the introduction of rotavirus vaccination programmes [2629]. The higher proportion of $\mathrm{G}_{2} \mathrm{P}[4]$ cases in the vaccine-era has coincided with an increase in the median age of cases, and a decline in cases among vaccine age-eligible age groups. These data may be interpreted in the context of varying strain fitness and partial heterotypic protection. Rotavirus $\mathrm{G}_{2} \mathrm{P}[4]$ genotypes may be displaced by other, better adapted or fitter genotypes such as $\mathrm{G}_{1} \mathrm{P}[8]$, particularly in an immunologically naive population. The association of $\mathrm{G}_{2} \mathrm{P}[4]$ with more frequent infection in older unvaccinated individuals who are likely to have had previous rotavirus infections suggests that the level of cross-protection against $\mathrm{G}_{2} \mathrm{P}[4]$ is not the same as that against the other genotype constellation 1 (Wa-like) strains (typically G1P[8], G3P[8], $\mathrm{G}_{4} \mathrm{P}[8], \mathrm{G}_{9} \mathrm{P}[8]$ and $\mathrm{G}_{12} \mathrm{P}[8]$ ]) [29].

In the vaccine era, the higher proportion of wildtype $G_{1} P[8]$ in infants $<6$ months of age compared with older age groups could potentially indicate that wild-type $G_{1} P[8]$ remains the most competitive strain when infecting immunologically naive populations. In the absence of a vaccine programme the competitive 


\section{FIGURE 3}

Age-stratified crude and adjusted multinomial odds ratios for genotypes occurring before and after rotavirus vaccine introduction, England, September 2006-August 2016 (full model; n=7,368)

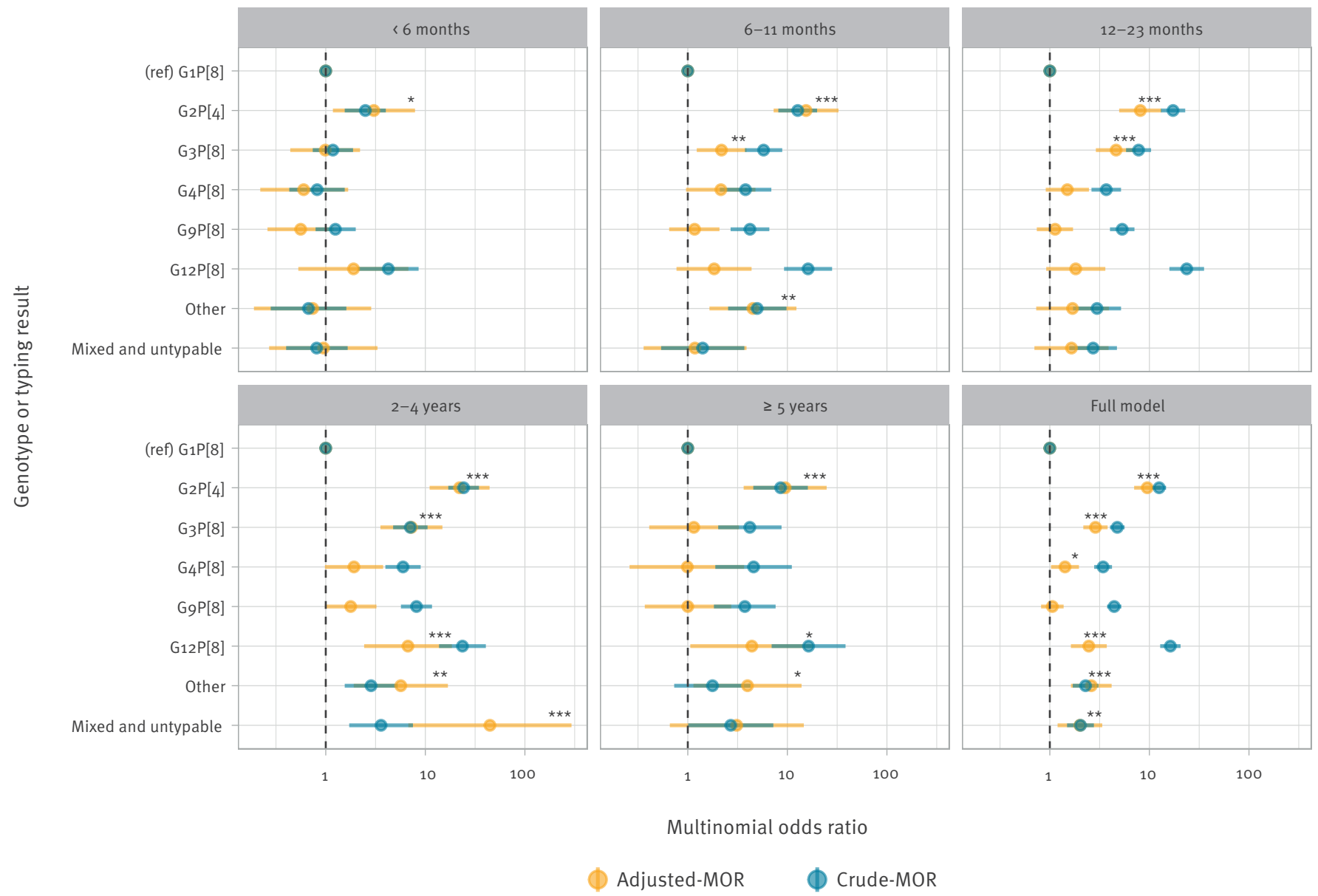

MOR: multinomial odds ratio; ref: reference.

MORs for vaccine era occurrence were estimated with multinomial logistic regression with the outcome variable genotype (G1P[8] as the reference group). Models were additionally adjusted for surveillance year (September-August) and season (and age group for full model). Specimens with unknown case age $(n=466)$ were excluded from the analysis. $\mathrm{G}_{1} \mathrm{P}[8]$ vaccine-derived strains $(\mathrm{n}=208)$ and $\mathrm{G}_{1} \mathrm{P}[8]$ - $\mathrm{unknown}$ (strains where it was unknown whether they were wild type or vaccine derived: $n=2$ ) were excluded from all models, under the assumption that the majority were detected in infants likely to have been vaccinated and were therefore not causative agent of the gastroenteritis symptoms. P values for adjusted multinomial odds ratios ${ }^{\star} p<0.05,{ }^{* \star} p<0.01,{ }^{* \star *} p<0.001$.

advantage of $\mathrm{G} 1 \mathrm{P}[8]$ strains appears to wane as infants become older and are exposed to repeated infections conferring protection through a varying degree of heterotypic immunity. The introduction of the monovalent rotavirus vaccine provides universal exposure to $\mathrm{G}_{1} \mathrm{P}[8]$ rotavirus among infants, with protection that is likely to be higher against homotypic strains than heterotypic ones, such as $\mathrm{G}_{2} \mathrm{P}[4]$, meaning that natural infection leading to disease is more likely to be caused by such heterotypic strains. This is further evidenced by the increase in rotavirus genotype diversity in samples from children aged 6 to 59 months.

The dominance of wild-type $\mathrm{G}_{1} \mathrm{P}[8]$ in the pre-vaccine period is also consistent with independent studies conducted in England during the late 1990 s and early 2000 s $[13,15,16]$. This adds strength to the evidence from this study that the decline in the relative proportion of wild-type $\mathrm{G}_{1} \mathrm{P}[8]$ and the relative rise of heterotypic strains such as $\mathrm{G}_{2} \mathrm{P}[4]$ in the vaccine-era is related to vaccine introduction rather than concurrent natural fluctuation. Furthermore, countries such as Belgium, Germany and Scotland introduced the Rotarix vaccine into their national childhood vaccination schedules in 2006, 2013 and 2013, respectively, and have all since seen an absolute decline in $\mathrm{G}_{1} \mathrm{P}[8]$, with $a$ rise in the proportion and absolute number of $\mathrm{G}_{2} \mathrm{P}[4]$ strain detections; Belgium reported slightly lower vaccine effectiveness against $\mathrm{G}_{2} \mathrm{P}[4]$ and Germany detected proportionally more $\mathrm{G}_{2} \mathrm{P}[4]$ genotypes in Rotarix-vaccinated children [30-33]. This is also true in lower income countries such as Malawi where point estimates for Rotarix vaccine effectiveness were lower against $\mathrm{G}_{2}$ strains than $\mathrm{G}_{1}$ strains ( $53 \%$ vs $82 \%$ ) [34]. 


\section{FIGURE 4}

Rotavirus genotype diversity measured using Shannon's index and Simpson's index of diversity, with 95\% confidence intervals, by age group and vaccine period, England, September 2006-August $2016(\mathrm{n}=7,128)$

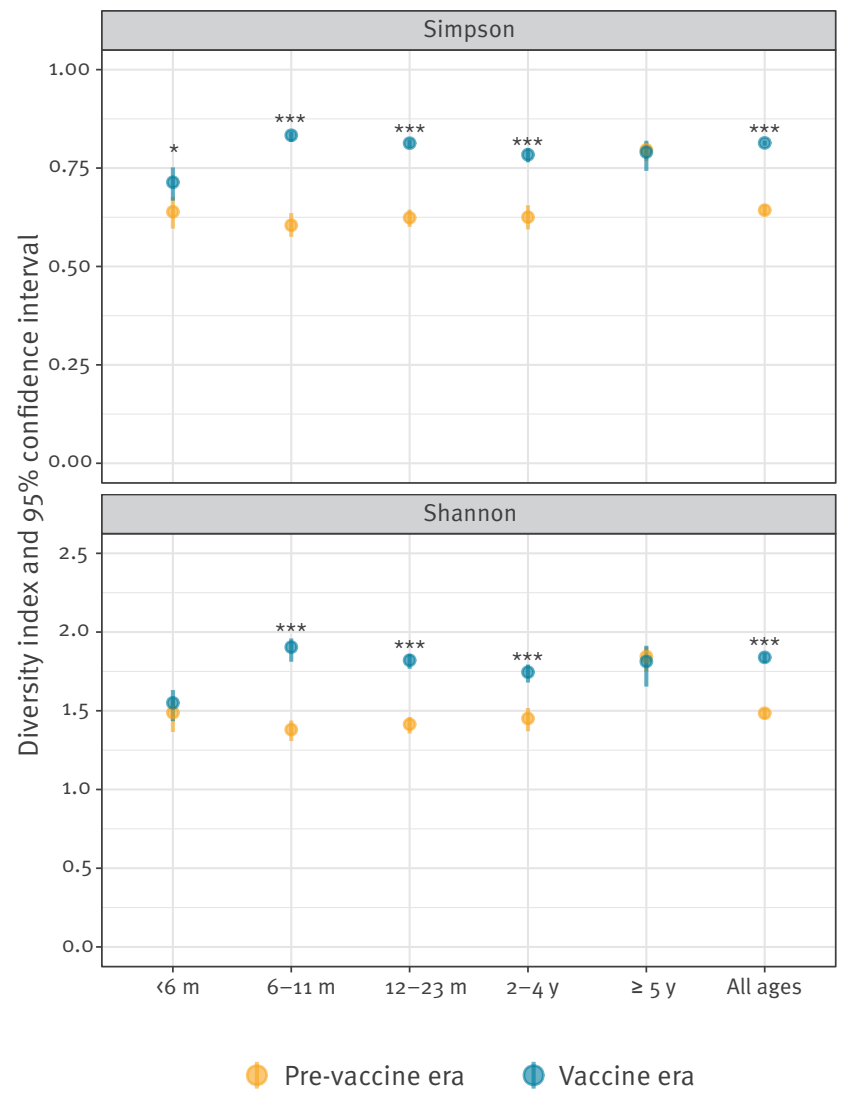

$\mathrm{m}$ : months, y: years.

A total of 466 specimens with unknown patient age and 240 mixed or untypable specimens were not considered, as only specimens with a recorded age and single typed rotavirus genotypes are included (G10P[4], G12P[10], G12P[4], G12P[6], G12P[8], G1P[4], $\mathrm{G}_{1} \mathrm{P}[5], \mathrm{G}_{1} \mathrm{P}[6], \mathrm{G}_{1} \mathrm{P}[8], \mathrm{G}_{2} \mathrm{P}[10], \mathrm{G}_{2} \mathrm{P}[4], \mathrm{G}_{2} \mathrm{P}[6], \mathrm{G}_{2} \mathrm{P}[8], \mathrm{G}_{2} \mathrm{P}[9]$ ], $\mathrm{G}_{3} P[14], \mathrm{G}_{3} P[3], \mathrm{G}_{3} P[4], \mathrm{G}_{3} P[6], \mathrm{G}_{3} P[8], \mathrm{G}_{3} P[9], \mathrm{G}_{4} P[4], \mathrm{G}_{4} P[8]$, G6P[14], G6P[9], G8P[14], G8P[4], G8P[8], G9P[4], G9P[6], G9P[8]). $\mathrm{G}_{1} \mathrm{P}[8]$ vaccine-derived strains $(n=208)$ as well as $\mathrm{G}_{1} \mathrm{P}[8]$-unknown (i.e. strains where it was unknown whether they were wild-type or vaccine derived; $n=2$ ) were excluded from the diversity analysis, under the assumption that the majority were detected in infants likely to have been vaccinated and were therefore not the causative agent of the gastroenteritis symptoms. $P$ values for a comparison between pre-vaccine and vaccine periods ${ }^{\star} p<0.05,{ }^{* \star} p<0.01$, $\star * \star p<0.001$.

The relative rise of $\mathrm{G}_{12} \mathrm{P}[8]$ in $2014 / 15$ across all age groups is likely to reflect natural seasonal fluctuations and global emergence rather than a force of vaccine selection. Belgium also experienced an increase in rotavirus cases with $\mathrm{G}_{12} \mathrm{P}$ [8] detection in 2014/15 [35]. Rapid increases and subsequent declines of $\mathrm{G} 12 \mathrm{P}[8]$ have been seen in other European countries; in $2011 / 12, G_{12} P[8]$ was the dominant strain in Spain; with the majority of detections occurring in the Gipuzkoa region of north eastern Spain; however, detections of $\mathrm{G} 12 \mathrm{P}[8]$ fell in 2012/13 [36]. There has also been independent introduction of $\mathrm{G} 12$ strains into Italy in recent years [37]. The emergence of $\mathrm{G}_{12}$ strains in countries both with and without rotavirus vaccination has been reported globally since 2004 [38-40].

In the pre-vaccine era, the predominant genotype $\mathrm{G}_{1} \mathrm{P}[8]$ declined with increasing age, intimating differences in homotypic and heterotypic immunity generated by natural infection over a person's life-course. It is, therefore, possible that the concept of 'antigenic sin' is applicable to rotavirus and vaccination. If this is the case, it may be expected that immunity to the homotypic strains may be sustained long-term even if circulation of $\mathrm{G}_{1} \mathrm{P}[8]$ rotaviruses is significantly reduced, through reinfections with other genotypes. This combination of factors poses the question as to whether vaccination will effectively mimic long-term protection previously generated through multiple natural infections and whether this could, in the future, result in a shift to milder disease caused by heterotypic strains in older vaccinated children.

In this study, we identified a number of cases with $\mathrm{G}_{1} \mathrm{P}[8]$ vaccine-derived strains. The majority of these were detected in vaccinated children aged 2 to 5 months and are likely not to be the cause of the symptoms. These children could be shedding vaccine strain after vaccination and illness is perhaps caused by other infectious pathogens or non-infectious aetiologies [41-43]. Furthermore, the high proportion of $\mathrm{G}_{1} \mathrm{P}$ [8] vaccine-derived strains may to some extent be the result of the increasing use of molecular methods in diagnostic laboratories across England; these methods will detect shedding of vaccine strain, potentially for months after vaccination, because of higher sensitivity compared with antigen detection methods [44,45].

The detection of $\mathrm{G}_{1} \mathrm{P}[8]$ vaccine-derived strains in older vaccine-eligible and vaccine-ineligible children would potentially suggest some horizontal transmission from vaccinated infants or persistent shedding in a vaccinated immunosuppressed child, such as those with severe combined immune deficiency [46]. A randomised placebo-controlled trial study in twins showed instances of horizontal transmission of vaccine strains between a vaccinated and unvaccinated twins but without gastroenteritis symptoms [47]. In the case of those $\mathrm{G}_{1} \mathrm{P}[8]$ vaccine-derived strains detected prevaccine introduction, this could be due to: vaccination in another country; privately accessed vaccination; and travel to or contact with children from countries where vaccination was available.

\section{Strengths and limitations}

Our analysis has benefited from England having one of the largest historic rotavirus surveillance systems in Europe. These data provide sufficient sample size year on year to detect genotypes with a prevalence of $\geq 1 \%$, allowing the analyses of the relative genotype prevalence and diversity presented. Importantly, this allowed us to adjust for seasonal trends and fluctuations in the models. We were also able to establish the 
vaccine status of cases with a detected $\mathrm{G}_{1} \mathrm{P}[8]$ vaccinederived strain.

However, there are limitations which need to be considered. Principally, it is important to be clear that the data included in this study are not representative of disease burden or incidence for the following reasons. Firstly, patients with acute gastroenteritis are advised not to seek medical help, because symptoms are usually self-limiting, particularly in older children and adults. Even if individuals seek medical attention, the National Institute of Clinical Excellent (NICE) guidance and UK guidance advise that testing for rotavirus is a clinical decision, recommended for children under 5 years of age with symptoms, particularly if symptoms are severe, prolonged or atypical $[48,49]$. Therefore, faecal specimens sent by clinicians for laboratory diagnostics are more likely to be from patients under 5 years of age with moderate to severe disease.

Secondly, even after identifying a rotavirus-positive sample, genotyping is reliant on the laboratory sending the specimen to the PHE VRD. Since 2013, all rotavirus-positive samples from vaccine eligible children have been actively followed up by PHE with the diagnostic laboratory. But, before vaccine introduction in 2013, genotype surveillance of rotavirus-positive samples was more passive and laboratory dependent. Furthermore, the sample size was calculated to detect rotavirus genotypes with a prevalence of $\geq 1 \%$ not for estimating rotavirus incidence.

Finally, from 2013 onwards, more laboratories were using molecular methods for diagnostics. As molecular techniques for rotavirus diagnosis are overly sensitive and less representative of symptomatic infection compared with antigen detection methods, it is possible that a greater proportion of samples were from cases where another infectious or non-infectious cause is responsible for the gastroenteritis symptoms. It would, therefore, be beneficial if samples could be tested for other gastrointestinal pathogens, especially in the case of $\mathrm{G}_{1} \mathrm{P}[8]$ vaccine-derived samples.

\section{Conclusions}

Rotavirus disease burden in England has greatly decreased since the introduction of rotavirus vaccination in 2013. This study shows that rotavirus genotype distribution and diversity has also changed in England since the introduction of vaccination with a large relative shift towards $\mathrm{G}_{2} \mathrm{P}[4], \mathrm{G}_{3} \mathrm{P}[8]$ and $\mathrm{G}_{12} \mathrm{P}[8]$. These changes will need continued surveillance, especially as the number and age of the vaccinated birth cohort's increase over the coming years. The results presented here and continued genotype surveillance across Europe through EuroRotaNet will help to inform whether future modifications to rotavirus vaccines may be needed. Ideally, a prospective birth cohort study would be used to assess both disease incidence and prevalence of rotavirus genotypes in symptomatic infections. Specifically, investigating how homotypic and heterotypic protection delivered by the vaccine compares to that induced by natural infection and in relation to age would be relevant.

\section{Acknowledgements}

We are indebted to all the physicians and microbiologists the help to collect clinical and microbiological data for rotavirus patients across the UK and to all EuroRotaNet members. We are also appreciative to, from Public Health England, Nick Andrews for his statistical comment and Charlotte Gower for providing vaccine histories.

Funding: Public Health England funded enhanced rotavirus surveillance from 2013. The authors received no financial support or other form of compensation related to the development of the manuscript. MIG receives support from The Wellcome Trust and the National Institute for Health Research Health Protection Research Unit in Gastrointestinal Infections at the University of Liverpool. DH was supported by the University of Liverpool.

Research approval and ethics: Data are anonymous and provided through EuroRotaNet and Public Health England as routine rotavirus strain surveillance; therefore, no ethical approval is required. Public Health England has approval, under Patient Information Advisory Group Section 60 of the Health and Social Care Act 2001, to process confidential information from patients for the purposes of monitoring the efficacy and safety of vaccination programmes.

\section{Conflict of interest}

Rotarix is developed and licensed by GlaxoSmithKline (GSK) Biologicals.

EuroRotaNet is funded by GlaxoSmithKline Biologicals (GSK) and Sanofi Pasteur, and Merck \& Co., Inc. (Kenilworth, NJ USA) after the closure of Sanofi Pasteur-MSD in December 2016. Both funders were provided the opportunity to review a version of this manuscript for factual accuracy but the authors are solely responsible for final content and interpretation.

Authors' contributions

$\mathrm{DH}$ contributed to study design, was responsible for data management and analyses and wrote the manuscript. DA contributed to study design, conducted the literature review and contributed to data collection. SN performed laboratory testing. SC and SL were responsible for the enhanced surveillance of the national rotavirus vaccination programme. RV contributed to study design and advised on analyses. MIG conceived of the study and contributed to data collection and analysis. All authors contributed to interpretation of the data, drafting the manuscript and final approval of the version to be published. No person or persons other than the authors listed have contributed significantly to the study or manuscript preparation.

\section{References}

1. Tate JE, Burton AH, Boschi-Pinto C, Steele AD, Duque J, Parashar UD, et al. . 2008 estimate of worldwide rotavirusassociated mortality in children younger than 5 years before the introduction of universal rotavirus vaccination programmes: a systematic review and meta-analysis. Lancet 
Infect Dis. 2012;12(2):136-41. https://doi.org/10.1016/S14733099(11)70253-5 PMID: 22030330

2. Harris JP, Jit M, Cooper D, Edmunds WJ. Evaluating rotavirus vaccination in England and Wales. Part I. Estimating the burden of disease. Vaccine. 2007;25(20):3962-70. https://doi. org/10.1016/j.vaccine.2007.02.072 PMID: 17395343

3. Tam CC, Rodrigues LC, Viviani L, Dodds JP, Evans MR, Hunter $P R$, et al. . Longitudinal study of infectious intestinal disease in the UK (IID2 study): incidence in the community and presenting to general practice. Gut. 2012;61(1):69-77. https://doi. org/10.1136/gut.2011.238386 PMID: 21708822

4. Iturriza-Gómara M, Cunliffe N. Rotavirus vaccine: a welcome addition to the immunisation schedule in the UK. BMJ. 2013;346(apr15 4):f2347. https://doi.org/10.1136/bmj.f2347 PMID: 23587749

5. Public Health England. National rotavirus immunisation programme: preliminary data for England, February 2016 to July 2016. Health Prot Rep Wkly Rep. 2016;10:1-6.

6. Ruiz-Palacios GM, Pérez-Schael I, Velázquez FR, Abate H, Breuer T, Clemens SC, et al. Human Rotavirus Vaccine Study Group. Safety and efficacy of an attenuated vaccine against severe rotavirus gastroenteritis. N Engl J Med. 2006;354(1):11 22. https://doi.org/10.1056/NEJMoa052434 PMID: 16394298

7. De Vos B, Han HH, Bouckenooghe A, Debrus S, Gillard P, Ward $R$, et al. Live attenuated human rotavirus vaccine, RIX 4414, provides clinical protection in infants against rotavirus strains with and without shared $G$ and $P$ genotypes: integrated analysis of randomized controlled trials. Pediatr Infect Dis J. 2009;28(4):261-6. https://doi.org/10.1097/ INF.ob013e3181907177 PMID: 19289978

8. Atchison CJ, Stowe J, Andrews N, Collins S, Allen DJ, Nawaz S, et al. Rapid Declines in Age Group-Specific Rotavirus Infection and Acute Gastroenteritis Among Vaccinated and Unvaccinated Individuals Within 1 Year of Rotavirus Vaccine Introduction in England and Wales. J Infect Dis. 2016;213(2):243-9. https:// doi.org/10.1093/infdis/jiv398 PMID: 26232438

9. Bawa Z, Elliot AJ, Morbey RA, Ladhani S, Cunliffe NA, O’Brien SJ, et al. Assessing the Likely Impact of a Rotavirus Vaccination Program in England: The Contribution of Syndromic Surveillance. Clin Infect Dis. 2015;61(1):77-85. https://doi. org/10.1093/cid/civ264 PMID: 25828997

10. Hungerford D, Read JM, Cooke RPD, Vivancos R, IturrizaGómara M, Allen DJ, et al. Early impact of rotavirus vaccination in a large paediatric hospital in the UK. J Hosp Infect. 2016;93(2):117-20. https://doi.org/10.1016/j.jhin.2015.12.010 PMID: 26876744

11. Hungerford D, Vivancos R, Read IM, Iturriza-Gómara $M$, French N, Cunliffe NA. Rotavirus vaccine impact and socioeconomic deprivation: an interrupted time-series analysis of gastrointestinal disease outcomes across primary and secondary care in the UK. BMC Med. 2018;16(1):10. https:// doi.org/10.1186/s12916-017-0989-Z PMID: 29375036

12. Matthijnssens J, Ciarlet M, Rahman M, Attoui H, Bányai K, Estes MK, et al. Recommendations for the classification of group A rotaviruses using all 11 genomic RNA segments. Arch Virol. 2008;153(8):1621-9. https://doi.org/10.1007/s00705008-0155-1 PMID: 18604469

13. Iturriza-Gómara M, Green J, Brown DW, Ramsay M, Desselberger U, Gray JJ. Molecular epidemiology of human group A rotavirus infections in the United Kingdom between 1995 and 1998. J Clin Microbiol. 2000;38(12):4394-401. PMID: 11101570

14. Iturriza-Gómara M, Dallman T, Bányai K, Böttiger B, Buesa J, Diedrich S, et al. Rotavirus genotypes co-circulating in Europe between 2006 and 2009 as determined by EuroRotaNet, a pan European collaborative strain surveillance network. Epidemiol Infect. 2011;139(6):895-909. https://doi.org/10.1017/ S0950268810001810 PMID: 20707941

15. Iturriza-Gómara M, Elliot AJ, Dockery C, Fleming DM, Gray J. Structured surveillance of infectious intestinal disease in pre-school children in the community: 'The Nappy Study'. Epidemiol Infect. 2009;137(7):922-31. https://doi.org/10.1017/ So950268808001556 PMID: 19017426

16. Iturriza Gómara M, Simpson R, Perault AM, Redpath C, Lorgelly $P$, Joshi $D$, et al. Structured surveillance of infantile gastroenteritis in East Anglia, UK: incidence of infection with common viral gastroenteric pathogens. Epidemiol Infect. 2008;136(1):23-33. https://doi.org/10.1017/ So950268807008059 PMID: 17313697

17. Hungerford D, Vivancos R, Read JM, Pitzer VE, Cunliffe N, French N, et al. EuroRotaNet network members. In-season and out-of-season variation of rotavirus genotype distribution and age of infection across 12 European countries before the introduction of routine vaccination, 2007/08 to 2012/13. Euro Surveill. 2016;21(2):30106. https://doi.org/10.2807/1560-7917. ES.2016.21.2.30106 PMID: 26794258
18. EuroRotaNet. EuroRotaNet n.d. [Accessed 13 Apr 2018]. Available from: http://www.eurorota.net/

19. Iturriza-Gómara M, Dallman T, Bányai K, Böttiger B, Buesa J, Diedrich S, et al. Rotavirus surveillance in europe, 2005-2008: web-enabled reporting and real-time analysis of genotyping and epidemiological data. J Infect Dis. 2009;200(s1) Suppl 1;S215-21. https://doi.org/10.1086/605049 PMID: 19821712

20. EuroRotaNet. Rotavirus Detection and Typing. UK: EuroRotaNet; 2009.

21. Gautam R, Esona MD, Mijatovic-Rustempasic S, Ian Tam K, Gentsch JR, Bowen MD. Real-time RT-PCR assays to differentiate wild-type group A rotavirus strains from Rotarix $(\circledR)$ and RotaTeq $(\circledR)$ vaccine strains in stool samples. Hum Vaccin Immunother. 2014;10(3):767-77. https://doi. org/10.4161/hv.27388 PMID: 24342877

22. R Core Team. R: A Language and Environment for Statistical Computing. Vienna, Austria: R Foundation for Statistical Computing; 2016.

23. Legendre P, Legendre LFJ. Numerical Ecology, Volume 24, Third Edition. 3 edition. Amsterdam: Elsevier; 2012.

24. Oksanen J, Blanchet FG, Kindt R, Legendre P, Minchin PR, O'Hara RB, et al. vegan: Community Ecology Package. 2013.

25. Gardener M. Community ecology : analytical methods using R and Excel®. Exeter: Pelagic Publishing; 2014.

26. Griffin DD, Fletcher M, Levy ME, Ching-Lee M, Nogami R, Edwards $L$, et al. Outbreaks of adult gastroenteritis traced to a single genotype of rotavirus. J Infect Dis. 2002;185(10):1502-5. https://doi.org/10.1086/340218 PMID: 11992287

27. Mikami T, Nakagomi T, Tsutsui R, Ishikawa K, Onodera Y, Arisawa $\mathrm{K}$, et al. An outbreak of gastroenteritis during school trip caused by serotype $\mathrm{G} 2$ group A rotavirus. J Med Virol. 2004;73(3):460-4. https://doi.org/10.1002/jmv.20112 PMID: 15170643

28. Antunes H, Afonso A, Iturriza M, Martinho I, Ribeiro C, Rocha $\mathrm{S}$, et al. G2P[4] the most prevalent rotavirus genotype in 2007 winter season in an European non-vaccinated population. J Clin Virol. 2009;45(1):76-8. https://doi.org/10.1016/j. jcv.2009.03.010 PMID: 19375980

29. Cardemil CV, Cortese MM, Medina-Marino A, Jasuja S, Desai $\mathrm{R}$, Leung J, et al. Rotavirus Investigation Team. Two rotavirus outbreaks caused by genotype $\mathrm{G}_{2} \mathrm{P}[4]$ at large retirement communities: cohort studies. Ann Intern Med. 2012;157(9):62131. https://doi.org/10.7326/o003-4819-157-9-20121106000006 PMID: 23128862

30. Braeckman T, Van Herck K, Meyer N, Pirçon J-Y, SorianoGabarró M, Heylen E, et al. RotaBel Study Group. Effectiveness of rotavirus vaccination in prevention of hospital admissions for rotavirus gastroenteritis among young children in Belgium: case-control study. BMJ. 2012;345(augo8 1):e4752. https:// doi.org/10.1136/bmj.e4752 PMID: 22875947

31. Matthijnssens J, Zeller M, Heylen E, De Coster S, Vercauteren J, Braeckman T, et al. RotaBel study group. Higher proportion of $\mathrm{G}_{2} \mathrm{P}[4]$ rotaviruses in vaccinated hospitalized cases compared with unvaccinated hospitalized cases, despite high vaccine effectiveness against heterotypic $\mathrm{G}_{2} \mathrm{P}[4]$ rotaviruses. Clin Microbiol Infect. 2014;20(10):0702-10. https://doi. org/10.1111/1469-0691.12612 PMID: 24580887

32. Mukhopadhya I, Murdoch H, Berry S, Hunt A, Iturriza-Gomara $M$, Smith-Palmer A, et al. Changing molecular epidemiology of rotavirus infection after introduction of monovalent rotavirus vaccination in Scotland. Vaccine. 2017;35(1):156-63. https:// doi.org/10.1016/j.vaccine.2016.11.028 PMID: 27876201

33. Adlhoch C, Hoehne M, Littmann M, Marques AM, Lerche A, Dehnert $M$, et al. Rotavirus vaccine effectiveness and casecontrol study on risk factors for breakthrough infections in Germany, 2010-2011. Pediatr Infect Dis J. 2013;32(2):e829. https://doi.org/10.1097/INF.ob013e3182720b71 PMID: 23334342

34. Bar-Zeev N, Kapanda L, Tate JE, Jere KC, Iturriza-Gomara M, Nakagomi O, et al. VacSurv Consortium. Effectiveness of a monovalent rotavirus vaccine in infants in Malawi after programmatic roll-out: an observational and case-control study. Lancet Infect Dis. 2015;15(4):422-8. https://doi. org/10.1016/S1473-3099(14)71060-6 PMID: 25638521

35. EuroRotaNet. EuroRotaNet: annual report 2016. Liverpool, UK: University of Liverpool; 2017.

36. Cilla G, Montes M, Gomariz M, Alkorta M, Iturzaeta A, PerezYarza EG, et al. Rotavirus genotypes in children in the Basque Country (North of Spain): rapid and intense emergence of the G12 [P8] genotype. Epidemiol Infect. 2013;141(4):868-74 https://doi.org/10.1017/So950268812001306 PMID: 22873952

37. Giammanco GM, Bonura F, DI Bernardo F, Cascio A, Ferrera G, Dones $P$, et al. Introduction and prolonged circulation of $\mathrm{G}_{12}$ rotaviruses in Sicily. Epidemiol Infect. 2016;144(9):1943-50. https://doi.org/10.1017/S0950268815003258 PMID: 26743189 
38. Dóró R, László B, Martella V, Leshem E, Gentsch J, Parashar $U$, et al. Review of global rotavirus strain prevalence data from six years post vaccine licensure surveillance: is there evidence of strain selection from vaccine pressure? Infect Genet Evol. 2014;28:446-61. https://doi.org/10.1016/j. meegid.2014.08.017 PMID: 25224179

39. Bányai K, László B, Duque J, Steele AD, Nelson EAS, Gentsch JR, et al. Systematic review of regional and temporal trends in global rotavirus strain diversity in the pre rotavirus vaccine era: insights for understanding the impact of rotavirus vaccination programs. Vaccine. 2012;30(Suppl 1):A122-30. https://doi.org/10.1016/j.vaccine.2011.09.111 PMID: 22520121

40. Luchs A, Cilli A, Morillo SG, de Souza Gregório D, Farias de Souza KA, Vieira HR, et al. Detection of the emerging rotavirus $\mathrm{G} 12 \mathrm{P}[8]$ genotype at high frequency in brazil in 2014: Successive replacement of predominant strains after vaccine introduction. Acta Trop. 2016;156:87-94. https://doi. org/10.1016/j.actatropica.2015.12.008 PMID: 26748357

41. Hsieh Y-C, Wu F-T, Hsiung CA, Wu H-S, Chang K-Y, Huang Y-C. Comparison of virus shedding after lived attenuated and pentavalent reassortant rotavirus vaccine. Vaccine. 2014;32(10):1199-204. https://doi.org/10.1016/j. vaccine.2013.08.041 PMID: 24076325

42. Kaneko M, Takanashi S, Thongprachum A, Hanaoka N, Fujimoto $\mathrm{T}$, Nagasawa K, et al. Identification of vaccine-derived rotavirus strains in children with acute gastroenteritis in Japan, 20122015. PLoS One. 2017;12(9):e0184067. https://doi.org/10.1371/ journal.pone.0184067 PMID: 28902863

43. Boom JA, Sahni LC, Payne DC, Gautam R, Lyde F, MijatovicRustempasic S, et al. Symptomatic infection and detection of vaccine and vaccine-reassortant rotavirus strains in 5 children: a case series. J Infect Dis. 2012;206(8):1275-9. https://doi. org/10.1093/infdis/jis490 PMID: 22872730

44. Bennett A, Bar-Zeev N, Jere KC, Tate JE, Parashar UD, Nakagomi 0 , et al. Determination of a Viral Load Threshold To Distinguish Symptomatic versus Asymptomatic Rotavirus Infection in a High-Disease-Burden African Population. J Clin Microbiol. 2015;53(6):1951-4. https://doi.org/10.1128/JCM.00875-15 PMID: 25854480

45. Mijatovic-Rustempasic S, Immergluck LC, Parker TC, Laghaie E, Mohammed A, McFadden T, et al. Shedding of porcine circovirus type 1 DNA and rotavirus RNA by infants vaccinated with Rotarix ${ }^{\circledR}$. Hum Vaccin Immunother. 2017;13(4):92835. https://doi.org/10.1080/21645515.2016.1255388 PMID: 27936349

46. Kaplon J, Cros G, Ambert-Balay K, Leruez-Ville M, Chomton M, Fremy $C$, et al. Rotavirus vaccine virus shedding, viremia and clearance in infants with severe combined immune deficiency. Pediatr Infect Dis J. 2015;34(3):326-8. https://doi.org/10.1097/ INF.0000000000000560 PMID: 25742082

47. Rivera L, Peña LM, Stainier I, Gillard P, Cheuvart B, Smolenov $\mathrm{I}$, et al. Horizontal transmission of a human rotavirus vaccine strain--a randomized, placebo-controlled study in twins. Vaccine. 2011;29(51):9508-13. https://doi.org/10.1016/j. vaccine.2011.10.015 PMID: 22008819

48. National Institute for Health and Care Excellence. Diarrhoea and vomiting caused by gastroenteritis in under $5 \mathrm{~s}$ : diagnosis and management. Manchester, UK: National Institute for Health and Clinical Excellence; 2009.

49. Public Health England. UK Standards for Microbiology Investigations S 7: Gastroenteritis and diarrhoea. London: Public Health England; 2013.

\section{License and copyright}

This is an open-access article distributed under the terms of the Creative Commons Attribution (CC BY 4.0) Licence. You may share and adapt the material, but must give appropriate credit to the source, provide a link to the licence, and indicate if changes were made.

This article is copyright of the authors or their affiliated institutions, 2019. 